\title{
EFFECT OF NITROGEN FERTILIZATION REGIMES ON SEED YIELD AND OIL PERCENTAGE OF SOME CANOLA CULTIVARS
}

\author{
Maha S.M. El-Maleh, Abd El-Fattah H. Belal and Eman I. El- Sarag \\ Dept. Plant Prod., Fac. Environ. Agric. Sci., El- Arish, Suez Canal Univ., Egypt
}

\begin{abstract}
A field experiment was carried out at the Experimental Farm, Faculty of Environmental Agricultural Sciences, Rafah, North Sinai Governorate, Suez Canal University, during two successive winter seasons 2011/2012 and 2012/2013; to study the response of 5 canola cultivars to nitrogen bio-chemical fertilization regimes in respect to yield and yield components under North Sinai conditions. Application of nitrogen fertilizer $\mathrm{T}_{1}(100 \%$ mineral) significantly increased yield/plant, seed yield $\mathrm{kg} / \mathrm{fed}$. and oil yield, while, $(75 \%$ mineral) and (50\% mineral) gave the highest 1000 -seed weight at both seasons. Serw 4 with $100 \%$ mineral nitrogen fertilization regimes gave the highest seed yield per plot and high oil percentage. Generally, it could be concluded that the canola cultivar Serw 4 and $100 \%$ mineral nitrogen fertilization regime gave the heighst yield and can be recommended under North Sinai conditions.
\end{abstract}

Key words: Canola, Brassica napus, canola cultivars, nitrogen fertilizations, Biofertilizer, Azotobacter, $\mathrm{N}$-fixing bacteria.

\section{INTRODUCTION}

Canola (Brassica napus L.) is one of the main oil crops in many countries especially in Canada, European Union and USA, but in Egypt it is still uncommon as oil crop. Canola has a relatively high requirement of nitrogen, where the content of this nutrient in seeds and plant tissues is greater than in most grain crops. Addition of $60 \mathrm{~kg} \mathrm{~N} / \mathrm{fed}$ under Egyptian conditions increased its yield (Kandil, 1984). Elewa et al. (2014) showed that Wan 25 variety was superiorin seed yield and yield components than the other. Bagheri et al. (2011) Moghadamet al. (2011).

Many investigators reported that increasing nitrogen fertilizer levels significantly increased most of yield components and seed oil percent (Afridiet al., 2002; Ali and
Hassan, 2002 and Khan et al., 2002: Abd El-Motaleb and Gomma, 2004 and AlBarrak (2006). They concluded that seed oil content decreased with increasing nitrogen level.

\section{MATREALS AND METHODS}

A field experiment was carried out at the Farm of the Faculty of Environmental Agricultural Sciences, Rafah, Suez Canal University, North Sinai Governorate on canola (Brassica napus L.) during 2011/ 2012 and the 2012/2013 winter seasons. The experiment was laid out in split-plot randomized complete block design with three replications.

The main plots were devoted to the five canola cultivars (Serw4, Pactol, Sakha 1 , $\mathrm{Sakha}_{2}$ and $\mathrm{Sakha}_{3}$ ). While, the sub - plots 
were devoted to 5 nitrogen bio-chemical fertilization regimes $\left(T_{1}, T_{2}, T_{3}, T_{4}\right.$ and $\left.T_{5}\right)$ calculated from plant needs of nitrogen $\mathrm{N} /$ fed. from (Urea 46\%) and commercial fertilizer (Biogen viz. Azotobacter) which give $25 \%$ nitrogen needs as a biofertilizer source.

Plot area was $12 \mathrm{~m}^{2}$ (1/350 fed.) rows 20 $\mathrm{m}$ length and $0.6 \mathrm{~m}$ width with $30 \mathrm{~cm}$ within row. Cultivars were sown on $30^{\text {th }}$ and $17^{\text {th }}$ October in the first and second seasons. The plants were thinned twice, the $1^{\text {st }}$ at 30 days after planting (DAP), where, 3-4 plants per hill was left and the $2^{\text {nd }}$ at 45 DAP, where, one plant per hill was left. Drip irrigation system $(4.0 \mathrm{~L} / \mathrm{hr}$ for each dripper) with underground saline water (3500 ppm) pumped from a well was used. The experimental site was irrigated immediately after planting, then irrigation every 2 days till 45 days from sowing.

Thereafter, irrigation every 7 days was applied. The physical and chemical analyses of experimental soil site were determined according to Richard (1954) as shown in Table 2. The organic fertilization was applied at the rate of 20 $\mathrm{m}^{3} /$ fed., while phosphorus fertilizer was applied in the form of single superphosphate $\left(15.5 \% \mathrm{P}_{2} \mathrm{O}_{5}\right)$ at the rate of $200 \mathrm{~kg} / \mathrm{fed}$., during land preparation.

At harvesting time $\left(10^{\text {th }}\right.$ and $20^{\text {th }}$ April at the first and second seasons), ten plants were taken from each sub-plot to determine the yield attributes, while, plants of one square meter from each treatment were taken for determining the seed yield (kg/fed.):

1. Seed yield per plant (g/plant).

2. Seed yield (kg/fed.) was computed according to seed yield (plot/g).

3. Seed oil yield $(\mathrm{kg} / \mathrm{fed}$.) was computed according to seed yield ( $\mathrm{kg} / \mathrm{fed}$.) and oil percentage.

Five grams from fresh seeds were taken at harvesting time from each plot. Each sample was dried in an electric oven $\left(70^{\circ} \mathrm{C}\right)$ till a constant weight, then milled to a fine powder. Seed oil content was estimated according to A.O.A.C. (1990) using Soxhelt apparatus and petroleum ether as a solvent, and then oil percentage was calculated on dry weight basis. Data of the two seasons were subjected to proper statistical analysis of variance (Snedecor and Cochran, 1990) using M-STATC program. Mean values were compared at $\mathrm{P}<0.05$ using the multiple range test (Duncan, 1955).

\section{RESULTS AND DISCUSSION}

\section{Effect of canola cultivars}

As shown in Table 3, seed yield/plant was significantly affected due to varietal differences cultivars in the both seasons.Serw 4 , produced the highest seed yield per plant in both seasons (12.84 and $21.45 \mathrm{~g}$ ), while low seed yield/plant was observed in each of $\mathrm{Sakha}_{2}$ and $\mathrm{Sakha}_{3}$ in first season as well as Sakha ${ }_{1}$, Sakha $_{2}$ and Sakha $_{3}$ in second season. The increase in seed yield per plant in $\mathrm{Serw}_{4}$ may be attributed to the increase in plant height, number of racemes and also number of siliqua than the other cultivars. Similar results were reported by Mekki (2007 and 2013).

Seed yield $\mathrm{kg} / \mathrm{fed}$, was significantly affected by canola cultivars, whereas the differences among the five cultivars under study were significant. Serw 4 produced higher seed yield $\mathrm{kg} / \mathrm{fed}$., compared to the other cultivars. Such increases amounted $27.41 \%$ and $36.36 \%$ compared with $\mathrm{Sakha}_{3}$ at both seasons (Table 3). However, canola cultivars $\mathrm{Sakha}_{1}, \mathrm{Sakha}_{2}$ and $\mathrm{Sakha}_{3}$ gave the minimum seed yield $\mathrm{kg} /$ fed., in comparison with the other two cultivars.

This increase may be due to the increase in number of racemes, number of siliqua per plant, seed yield per plant, 1000-seed weight of $\mathrm{Serw}_{4}$ cultivar which reflected to increase the seed yield. Similar results were reported by Elewa et al. (2014). Regarding 
Sinai Journal of Applied Sciences (ISSN: 2314-6079) Vol. (4) Is.:(3), Dec., 2015

Table (1): Nitrogen fertilization types, rates and time of adding.

\begin{tabular}{|c|c|c|}
\hline Treatments & $\begin{array}{l}\text { Types } \\
\text { Mineral }\end{array}$ & Rate and time of adding \\
\hline $\mathbf{T}_{1}$ & $\begin{array}{c}100 \% \\
1.39 \mathrm{~kg}\end{array}$ & $\begin{array}{l}\text { Equal amounts after thinning + at beginning of } \\
\text { flowering on all nitrogen fertilization regimes. }\end{array}$ \\
\hline $\mathbf{T}_{2}$ & $\begin{array}{c}75 \% \\
1.04 \mathrm{~kg}\end{array}$ & One amount before seeding. \\
\hline $\mathbf{T}_{3}$ & $\begin{array}{c}50 \% \\
0.659 \mathrm{~kg}\end{array}$ & 2 amounts, before seeding + at thinning. \\
\hline $\mathbf{T}_{4}$ & $\begin{array}{c}25 \% \\
0.347 \mathrm{~kg}\end{array}$ & $\begin{array}{l}3 \text { amounts, before seeding }+ \text { at thinning }+ \text { at beginning } \\
\text { of flowering. }\end{array}$ \\
\hline $\mathbf{T}_{5}$ & $0 \%$ & \\
\hline
\end{tabular}

Table (2): Physical and chemical properties of the experimental soil site during the two seasons.

\begin{tabular}{|c|c|c|c|c|c|c|c|}
\hline $\begin{array}{c}\text { Soil } \\
\text { Properties }\end{array}$ & $\begin{array}{l}\text { Clay } \\
(\%)\end{array}$ & $\begin{array}{c}\text { Loam } \\
(\%)\end{array}$ & $\begin{array}{l}\text { Sand } \\
(\%)\end{array}$ & Texture & $\begin{array}{l}\text { Organic } \\
\text { matter\% }\end{array}$ & pH & $\begin{array}{c}\mathrm{EC} \\
(\mathrm{ds} / \mathrm{m} \\
1: 5)\end{array}$ \\
\hline Value & 0.40 & 3.50 & 96.10 & Sand & 1.15 & 7.03 & 3.84 \\
\hline \multirow[t]{2}{*}{$\begin{array}{l}\text { Cations } \\
(\text { meq/l) }\end{array}$} & $\mathrm{Ca}^{++}$ & $\mathrm{Mg}^{++}$ & $\mathrm{Na}^{+}$ & $\mathrm{K}^{+}$ & & & \\
\hline & 10.00 & 11.00 & 21.00 & 2.00 & & & \\
\hline \multirow[t]{2}{*}{$\begin{array}{l}\text { Anions } \\
\text { (meq/l) }\end{array}$} & $\mathrm{HCO}_{3}^{-}$ & $\mathrm{Cl}^{-}$ & $\mathrm{SO}_{4}^{--}$ & & & & \\
\hline & 6.60 & 26.40 & 11.00 & & & & \\
\hline
\end{tabular}


to the oil yield it was scientifically different in the performance of cultivars. Therefore, $\mathrm{Sakha}_{3}$ had the lowest oil yield/fed. The reduction on oil yield of Sakha 3 mainly due to the decreased on their seed yield $\mathrm{kg} / \mathrm{fed}$. (Table 3). Performance of Serw 4 had the highest oil yield due to the increase in their seed yield $\mathrm{kg} / \mathrm{fed}$.

These results may refer to genetically difference between cultivars. These results are similar with those obtained by Moghadam et al. (2011).

\section{Seed oil percentage}

Data in Table (3) shows the significant effect at $p<0.01$ of performance of canola cultivars on seed oil percentage at the both seasons. Serw 4 cultivar surpassed the other cultivars regarding seed oil (\%), Pactol ,Sakha ${ }_{1}$, Sakha $_{2}$ and Sakha $_{3}$ canola cultivars had insignificant differences seed oil (\%) among each other. These results depending on climatic conditions under grown $B$. napus. But there are slightly differences on performance of cultivars in oil seed (\%) at the second season. Whease $\mathrm{Serw}_{4}$ cultivar ranked first and Pactol ranked second, then followed by $\mathrm{Sakha}_{1}, \mathrm{Sakha}_{2}$ and $\mathrm{Sakha}_{3}$ without significant differences among Sakha cultivars.

This may refer to the fact that accumulation of fat take place during the development of storage tissue. Fat increase in quality and in concentration is probably due to transformation of sugar to fat in the seed from leaves.

On the other hand $\mathrm{Serw}_{4}$ and Pactol gave the highest value of plant height, number of racemes which may reflected on the increase of carbohydrates at such plants which consequently may encourage the transformation of carbohydrates to fat.

This due to the stability of oil percentage in canola cultivars. These results go in harmony with those obtained by Zarei $\boldsymbol{e t}$ al. (2010), Ali et al. (2011), Sayed et al. (2011).

\section{Effect of nitrogen fertilization regimes}

Nitrogen fertilizer is the most important element for crop growth and high yield with good quality. Seed yield and yield attributes were increased by increasing nitrogen levels.

The excessive use of nitrogen fertilizer has generated several environmental problems. Some of these problems can be solved by using bio-fertilizers which have natural benefits and safely to environment. The bio-fertilizers provide nutrients to the plants and maintain soil structure. It has been revealed that the effect of nitrogen fixation induced by nitrogen fixers is not only significant for legumes, but also nonlegumes.

The canola nitrogen requirement is the amount of nitrogen needed to produce high seed yield. Because of soils, weather conditions and agronomic practices that can influence nitrogen uptake, the nitrogen requirement determination is essential for maximum seed and oil yield of canola crop, especially under unfavorable growth conditions such as arid and semi-arid lands.

Results in Table 4 show that there were highly significant effects for nitrogen fertilization regimes on seeds yield/plant in the both studied seasons. Application of $\mathrm{T}_{1}$ increased seed yield per plant up to 15.33 and $25.00 \mathrm{~g}$ at the $1^{\text {st }}$ and $2^{\text {nd }}$ seasons. Applying $\mathrm{T}_{1}$ surpassed the other nitrogen regimes by $47.87 \%$ and $54.12 \%$ as compared with $\mathrm{T}_{5}$ at the both seasons.

This increase may be due to nitrogen general function in plant. Similar results were reported by Shahin et al. (2000) and Al-Barrak (2006).

Response of seed yield $\mathrm{kg} / \mathrm{fed}$., to nitrogen bio-chemical fertilization regimes was highly significant in canola crop (Table 4).

A gradual increase in seed yield $\mathrm{kg} / \mathrm{fed}$. was noticed with applying of $T_{1}(100 \%$ mineral). The increase in seed yield $\mathrm{kg} / \mathrm{fed}$. 
Table (3): Seed yield/plant (g), seed yield (kg/fed.), oil yield (kg/fed.) and seed oil percentage for the five studied cultivars at the two seasons.

\begin{tabular}{|c|c|c|c|c|c|c|c|c|}
\hline \multirow{3}{*}{$\begin{array}{l}\text { Canola } \\
\text { cultivars }\end{array}$} & \multicolumn{2}{|c|}{$\begin{array}{c}\text { Seed } \\
\text { yield/plant (g) }\end{array}$} & \multicolumn{2}{|c|}{$\begin{array}{l}\text { Seed yield } \\
\text { (kg/fed.) }\end{array}$} & \multicolumn{2}{|c|}{$\begin{array}{l}\text { Oil yield } \\
\text { (kg/fed.) }\end{array}$} & \multicolumn{2}{|c|}{$\begin{array}{c}\text { Seed oil } \\
\text { percentage }\end{array}$} \\
\hline & 2011/ & $2012 /$ & 2011/ & $2012 /$ & 2011/ & $2012 /$ & 2011/ & 2012/ \\
\hline & 2012 & 2013 & 2012 & 2013 & 2012 & 2013 & 2012 & 2013 \\
\hline Serw $_{4}$ & $12.84^{\mathrm{a}}$ & $21.45^{\mathrm{a}}$ & $292.11^{\mathrm{a}}$ & $487.98^{\mathrm{a}}$ & $127.59^{\mathrm{a}}$ & $207.24^{\mathrm{a}}$ & $43.68^{\mathrm{a}}$ & $42.47^{\mathrm{a}}$ \\
\hline Pactol & $12.63^{\mathrm{a}}$ & $17.65^{b}$ & $287.33^{\mathrm{a}}$ & $401.53^{b}$ & $119.58^{\mathrm{a}}$ & $153.90^{b}$ & $41.62^{b}$ & $38.33^{\mathrm{c}}$ \\
\hline Sakha $_{1}$ & $11.54^{\mathrm{a}}$ & $14.39^{c}$ & $262.53^{b}$ & $327.37^{\mathrm{c}}$ & $110.55^{b}$ & $131.55^{\mathrm{c}}$ & $42.11^{b}$ & $40.12^{b}$ \\
\hline Sakha $_{2}$ & $9.862^{b}$ & $14.36^{\mathrm{c}}$ & $224.36^{\mathrm{c}}$ & $326.69^{c}$ & $92.59^{c}$ & $131.34^{\mathrm{c}}$ & $41.27^{b}$ & $40.27^{b}$ \\
\hline Sakha $_{3}$ & $9.329^{b}$ & $13.65^{\mathrm{c}}$ & $212.03^{c}$ & $310.53^{c}$ & $86.88^{\mathrm{c}}$ & $125.17^{\mathrm{c}}$ & $40.98^{b}$ & $40.31^{b}$ \\
\hline Significant & $* *$ & $* *$ & $* *$ & $* *$ & $* *$ & $* *$ & $* *$ & ** \\
\hline
\end{tabular}

Table (4): Effect of nitrogen fertilization regimes on seed yield/plant (g), Seed yield (kg/fed.), Oil yield (kg/fed.)and seed oil percentage at the two seasons.

\begin{tabular}{ccccccccc}
\hline $\begin{array}{c}\text { Nitrogen } \\
\text { fertilization }\end{array}$ & \multicolumn{2}{c}{$\begin{array}{c}\text { Seed } \\
\text { yield/plant }(\mathbf{g})\end{array}$} & \multicolumn{2}{c}{$\begin{array}{c}\text { Seed yield } \\
\text { (kg/fed.) }\end{array}$} & \multicolumn{2}{c}{ Oil yield (kg/fed.) } & \multicolumn{2}{c}{$\begin{array}{c}\text { Seed oil } \\
\text { percentage }\end{array}$} \\
\cline { 2 - 9 } regimes & $\mathbf{2 0 1 1 /}$ & $\mathbf{2 0 1 2 /}$ & $\mathbf{2 0 1 1 /}$ & $\mathbf{2 0 1 2} /$ & $\mathbf{2 0 1 1 /}$ & $\mathbf{2 0 1 2} /$ & $\mathbf{2 0 1 1 /}$ & $\mathbf{2 0 1 2 /}$ \\
& $\mathbf{2 0 1 2}$ & $\mathbf{2 0 1 3}$ & $\mathbf{2 0 1 2}$ & $\mathbf{2 0 1 3}$ & $\mathbf{2 0 1 2}$ & $\mathbf{2 0 1 3}$ & $\mathbf{2 0 1 2}$ & $\mathbf{2 0 1 3}$ \\
\hline $\mathbf{T}_{\mathbf{1}}$ & $15.33^{\mathrm{a}}$ & $25.00^{\mathrm{a}}$ & $348.75^{\mathrm{a}}$ & $568.75^{\mathrm{a}}$ & $152.33^{\mathrm{a}}$ & $241.54^{\mathrm{a}}$ & $44.52^{\mathrm{a}}$ & $40.40^{\mathrm{ab}}$ \\
\hline $\mathbf{T}_{\mathbf{2}}$ & $13.15^{\mathrm{b}}$ & $17.73^{\mathrm{b}}$ & $299.16^{\mathrm{b}}$ & $403.35^{\mathrm{b}}$ & $124.51^{\mathrm{b}}$ & $154.60^{\mathrm{b}}$ & $43.96^{\mathrm{a}}$ & $40.55^{\mathrm{ab}}$ \\
\hline $\mathbf{T}_{3}$ & $11.36^{\mathrm{c}}$ & $14.58^{\mathrm{c}}$ & $258.44^{\mathrm{c}}$ & $331.69^{\mathrm{c}}$ & $108.82^{\mathrm{c}}$ & $133.07^{\mathrm{c}}$ & $41.49^{\mathrm{b}}$ & $40.80^{\mathrm{a}}$ \\
\hline $\mathbf{T}_{\mathbf{4}}$ & $8.37^{\mathrm{d}}$ & $12.72^{\mathrm{d}}$ & $190.19^{\mathrm{d}}$ & $289.38^{\mathrm{d}}$ & $80.96^{\mathrm{d}}$ & $116.53^{\mathrm{d}}$ & $41.33^{\mathrm{b}}$ & $40.06^{\mathrm{ab}}$ \\
\hline $\mathbf{T}_{\mathbf{5}}$ & $7.99^{\mathrm{d}}$ & $11.47^{\mathrm{d}}$ & $181.77^{\mathrm{d}}$ & $260.94^{\mathrm{e}}$ & $74.48^{\mathrm{d}}$ & $105.18^{\mathrm{e}}$ & $38.37^{\mathrm{c}}$ & $39.69^{\mathrm{b}}$ \\
\hline Significant & $* *$ & $* *$ & $* *$ & $* *$ & $* *$ & $* *$ & $* *$ & $* *$ \\
\hline
\end{tabular}


was estimated by $47.78 \%$ and $54.12 \%$ for $\mathrm{T}_{1}$ regime compared to $\mathrm{T}_{5}(0 \%$ chemical + biofertilizer) regime at the two seasons.

These increases in seed yield may be due to increase in vegetative growth stage, which reflected to the adequate supply of photosynthesis for formation of plant height at harvest, number of racemes/plant at harvest, number of siliqua/plant, seed yield/ plant, 1000 seed weight and seed yield per plot and development at seeds then it increased seed yield $\mathrm{kg} / \mathrm{fed}$.

So, biofertilizer leads to major negative affection between vegetative growth and yield production.

Similar finding have been reported by Afridiet al. (2000), Kappenet al. (2000), Sharief and Keshta (2000), Pennocket al. (2001), Ali and Hassan (2002), Khan et al. (2002), Abd El-Motaleb and Gomaa (2004) and El- Demrdash and Ali (2005).

Concerning, the oil yield ( $\mathrm{kg} / \mathrm{fed}$.), results in Table 4 shows that $T_{1}$ fertilization regime had high significant effect on oil yield $\mathrm{kg} /$ fed.

The increase in oil yield estimated by $51.10 \%$ and $56.45 \%$ in plants fertilized by $\mathrm{T}_{1}$ as a compared with $\mathrm{T}_{5}$ such increase in oil yield was mainly due to the increase of seed yield $\mathrm{kg} / \mathrm{fed}$. and also due to oil (\%).

These results are in line with those obtained by other studies Ahmed 2001 and Al-Barrak 2006.

\section{Seed oil percentage}

Data in Table 4 shows that there were significant effect of nitrogen fertilization regime on canola seed oil percentage at the $1^{\text {st }}$ and $2^{\text {nd }}$ seasons. The highest seed oil $\%$
(44.58 and $43.96 \%$ ) were obtained when canola was fertilized by $T_{1}$ as mineral fertilizer and $T_{2}$ at the $1^{\text {st }}$ season. However, $\mathrm{T}_{3}$ gave maximum seed oil percentage at the $2^{\text {nd }}$ season.

These results may due to the negative correlation between nitrogen fertilization, as a reason to increase protein content in seeds.

So, biofertilizer leads to major negative correlation between vegetative growth and yield attributes which resulted to decrease yield and oil (\%) in seeds. Khan et al. (2002) and Asghar et al. (2002) revealed that the seed oil content was decreased with increasing nitrogen rates

\section{Interaction}

The highest seed yield per plant (34.55 g) was produced by canola cv. Serw 4 with $100 \%$ mineral while, there is no significant differences in the first season (Table 5). The highest seed yield per feddan (395.16 and $786.01 \mathrm{~kg} / \mathrm{fed}$.) and oil yield (177.07 and $329.41 \mathrm{~kg} / \mathrm{fed}$.) were obtained by serw 4 cultivar with $100 \%$ mineral (Table 5).

Results in Table 6 cleared that the effect of interaction between canola cultivars and nitrogen fertilization treatments was significant on seed oil percentage.

The highest seed oil percentage was obtained by Pactol cultivar and $T_{2}$ in first season and sakha 3 and $\mathrm{T}_{1}$ in $2^{\text {nd }}$ season.

Generally, it could be recommended that fertilizing canola ( $\mathrm{Serw}_{4}$ cultivar) with $(100 \%$ mineral) increased the yield production, oil yield and oil percentage under North Sinai conditions. 
Table (5): Effect of canola cultivars and nitrogen fertilization regimes on yield and yield attributes (Seed yield/plant (g), Seed yield (kg/fed) and Oil yield (kg/fed) at the two seasons $(2011 / 2012 \& 2012 / 2013)$.

\begin{tabular}{|c|c|c|c|c|c|c|c|}
\hline \multirow[t]{3}{*}{ Cv. } & \multirow{3}{*}{$\begin{array}{l}\mathrm{N}- \\
\text { fertilization } \\
\text { regimes }\end{array}$} & \multicolumn{2}{|c|}{$\begin{array}{c}\text { Seed yield/ } \\
\text { plant(g) }\end{array}$} & \multicolumn{2}{|c|}{$\begin{array}{l}\text { Seed yield } \\
\text { (kg/fed) }\end{array}$} & \multicolumn{2}{|c|}{$\begin{array}{l}\text { Oil yield } \\
\text { (kg/fed) }\end{array}$} \\
\hline & & 2011/ & 2012/ & 2011/ & $2012 /$ & 2011/ & $2012 /$ \\
\hline & & 2012 & 2013 & 2012 & 2013 & 2012 & 2013 \\
\hline \multirow{5}{*}{ Serw $_{4}$} & $\mathbf{T}_{1}$ & 17.37 & $34.55^{\mathrm{a}}$ & $395.16^{\mathrm{a}}$ & $786.01^{\mathrm{a}}$ & $177.07^{\mathrm{a}}$ & $329.41^{\mathrm{a}}$ \\
\hline & $\mathbf{T}_{2}$ & 13.87 & $25.66^{b}$ & $315.54^{\mathrm{bcd}}$ & $315.54^{b}$ & $136.77^{\text {bcd }}$ & $234.96^{b}$ \\
\hline & $\mathbf{T}_{3}$ & 11.90 & $14.67^{\mathrm{e}-\mathrm{h}}$ & $270.72^{\text {def }}$ & $583.76^{c}$ & $123.17^{\mathrm{def}}$ & $126.25^{c}$ \\
\hline & $\mathbf{T}_{4}$ & 10.82 & $13.33^{\mathrm{e}-\mathrm{j}}$ & $246.15^{\mathrm{d}-\mathrm{g}}$ & $333.74^{\mathrm{efg}}$ & $112.53^{\mathrm{d}-\mathrm{g}}$ & $122.87^{\mathrm{efg}}$ \\
\hline & $\mathbf{T}_{5}$ & 10.25 & $9.05^{\mathrm{cd}}$ & $233.18^{\mathrm{fg}}$ & $205.88^{\mathrm{fgh}}$ & $101.03^{\mathrm{fg}}$ & $85.44^{\mathrm{fgh}}$ \\
\hline \multirow{5}{*}{ Pactol } & $\mathbf{T}_{1}$ & 15.30 & $28.44^{b}$ & $348.07^{b}$ & $647.01^{b}$ & $153.01^{b}$ & $273.87^{b}$ \\
\hline & $\mathbf{T}_{2}$ & 14.00 & $16.67^{\text {cde }}$ & $318.50^{b c}$ & $379.24^{\mathrm{e}}$ & $152.46^{\mathrm{bc}}$ & $148.41^{\mathrm{e}}$ \\
\hline & $\mathbf{T}_{3}$ & 12.40 & $15.72^{\text {def }}$ & $314.63^{\mathrm{bcd}}$ & $357.63^{\mathrm{e}}$ & $132.58^{\mathrm{bcd}}$ & $143.16^{\mathrm{e}}$ \\
\hline & $\mathbf{T}_{4}$ & 13.83 & $14.61^{\mathrm{e}-\mathrm{h}}$ & $282.10^{\text {efg }}$ & $332.37^{\mathrm{ef}}$ & $126.89^{\mathrm{efg}}$ & $134.04^{\mathrm{ef}}$ \\
\hline & $\mathbf{T}_{5}$ & 10.97 & $12.83^{\mathrm{e}-\mathrm{k}}$ & $249.56^{\mathrm{fg}}$ & $291.88^{\mathrm{hi}}$ & $101.39^{\mathrm{fg}}$ & $119.17^{\mathrm{hi}}$ \\
\hline \multirow{5}{*}{ Sakha $_{1}$} & $\mathbf{T}_{1}$ & 14.10 & $28.00^{b}$ & $341.25^{b c}$ & $637.00^{b}$ & $139.98^{b c}$ & $273.91^{b}$ \\
\hline & $\mathbf{T}_{2}$ & 13.30 & $15.00^{\mathrm{d}-\mathrm{h}}$ & $320.77^{\mathrm{bcd}}$ & $341.25^{\mathrm{efg}}$ & $136.74^{\mathrm{bcd}}$ & $133.87^{\mathrm{etg}}$ \\
\hline & $\mathbf{T}_{3}$ & 9.25 & $10.33^{\mathrm{ijk}}$ & $302.57^{\text {bcd }}$ & $227.50^{\mathrm{jk}}$ & $125.83^{\mathrm{bcd}}$ & $91.56^{\mathrm{k}}$ \\
\hline & $\mathbf{T}_{4}$ & 7.22 & $9.61^{\mathrm{jk}}$ & $210.00^{\mathrm{hi}}$ & $218.62^{\mathrm{k}}$ & $91.52^{\mathrm{hi}}$ & $89.65^{\mathrm{k}}$ \\
\hline & $\mathbf{T}_{5}$ & 7.00 & $9.00^{\mathrm{k}}$ & $164.25^{\mathrm{ij}}$ & $204.75^{\mathrm{k}}$ & $67.62^{\mathrm{ij}}$ & $82.92^{\mathrm{k}}$ \\
\hline \multirow{5}{*}{ Sakha $_{2}$} & $\mathbf{T}_{1}$ & 11.80 & $20.00^{c}$ & $268.45^{b c}$ & $455.00 \mathrm{~d}$ & $123.48^{b c}$ & $191.10^{\mathrm{d}}$ \\
\hline & $\mathbf{T}_{2}$ & 8.95 & $15.44^{\mathrm{d}-\mathrm{g}}$ & $203 .{ }^{61 c d e}$ & $351.26^{\mathrm{e}}$ & $81.95^{\text {cde }}$ & $141.10^{\mathrm{e}}$ \\
\hline & $\mathbf{T}_{3}$ & 8.55 & $13.44^{\mathrm{e}-\mathrm{J}}$ & $194.51^{\mathrm{gh}}$ & $305.76^{\mathrm{gh}}$ & $81.05^{\mathrm{gh}}$ & $117.71^{\mathrm{gh}}$ \\
\hline & $\mathbf{T}_{4}$ & 8.00 & $12.11^{\mathrm{f}-\mathrm{k}}$ & $182.00^{\mathrm{ij}}$ & $275.50^{\mathrm{hi}}$ & $69.34^{\mathrm{ij}}$ & $105.37^{\mathrm{hi}}$ \\
\hline & $\mathbf{T}_{5}$ & 7.13 & $10.83^{h-k}$ & $162.20^{j}$ & $246.38^{\mathrm{jk}}$ & $65.52^{j}$ & $101.92^{\mathrm{jk}}$ \\
\hline \multirow{5}{*}{ Sakha $_{3}$} & $T_{1}$ & 12.00 & $15.89^{\text {def }}$ & $273.00^{\mathrm{bc}}$ & $361.49^{d}$ & $111.93^{b c}$ & $155.80^{d}$ \\
\hline & $\mathbf{T}_{2}$ & 10.26 & $14.37^{\mathrm{e}-1}$ & $233.41^{\text {def }}$ & $326.91^{\mathrm{ef}}$ & $87.52^{\text {def }}$ & $135.36^{\mathrm{ef}}$ \\
\hline & $\mathbf{T}_{\mathbf{3}}$ & 5.25 & $14.00^{\mathrm{e}-1}$ & $119.43^{\mathrm{fg}}$ & $318.50^{\text {fgh }}$ & $39.41^{\mathrm{fg}}$ & $118.42^{\text {fgh }}$ \\
\hline & $\mathbf{T}_{4}$ & 4.00 & $12.62^{\mathrm{e}-\mathrm{k}}$ & $91.00^{j}$ & $287.10^{\mathrm{hi}}$ & $39.13^{j}$ & $111.96^{\mathrm{hi}}$ \\
\hline & $\mathbf{T}_{5}$ & 4.00 & $11.37^{\mathrm{g}-\mathrm{k}}$ & $91.00^{j}$ & $258.66^{\mathrm{ij}}$ & $33.97^{j}$ & $96.55^{\mathrm{ij}}$ \\
\hline \multicolumn{2}{|c|}{ Significant } & NS & $* *$ & $* *$ & $* *$ & $* *$ & $* *$ \\
\hline
\end{tabular}


Table (6): Effect of canola cultivars and nitrogen fertilization regimes on Seed oil (\%) at the two seasons.

\begin{tabular}{|c|c|c|c|}
\hline \multirow{2}{*}{ Cv. } & \multirow{2}{*}{$\begin{array}{l}\mathrm{N}- \\
\text { fertilization } \\
\text { regimes }\end{array}$} & \multicolumn{2}{|c|}{ Seed oil percentage } \\
\hline & & $2011 / 2012$ & $2012 / 2013$ \\
\hline \multirow{5}{*}{$\operatorname{Serw}_{4}$} & $\mathbf{T}_{1}$ & $44.81^{\mathrm{b}-\mathrm{f}}$ & $41.91^{\mathrm{a}-\mathrm{d}}$ \\
\hline & $\mathbf{T}_{2}$ & $43.25^{\mathrm{d}-\mathrm{h}}$ & $40.25^{\text {def }}$ \\
\hline & $\mathbf{T}_{3}$ & $45.50^{\mathrm{a}-\mathrm{e}}$ & $37.83^{\text {gh }}$ \\
\hline & $\mathbf{T}_{4}$ & $45.72^{\mathrm{a}-\mathrm{d}}$ & $40.52^{\text {cde }}$ \\
\hline & $\mathbf{T}_{5}$ & $43.33^{\mathrm{d}-\mathrm{h}}$ & $41.50^{\mathrm{a}-\mathrm{d}}$ \\
\hline \multirow{5}{*}{ Pactol } & $\mathbf{T}_{1}$ & $43.96^{\mathrm{c}-\mathrm{g}}$ & $42.33^{\mathrm{abc}}$ \\
\hline & $\mathbf{T}_{2}$ & $47.87^{\mathrm{a}}$ & $37.75^{\text {gh }}$ \\
\hline & $\mathbf{T}_{3}$ & $47.00^{\mathrm{ab}}$ & $41.50^{\mathrm{a}-\mathrm{d}}$ \\
\hline & $\mathbf{T}_{4}$ & $40.63^{1-k}$ & $40.33^{\mathrm{de}}$ \\
\hline & $\mathbf{T}_{5}$ & $40.33^{\mathrm{jk}}$ & $40.83^{\mathrm{b}-\mathrm{e}}$ \\
\hline \multirow{5}{*}{ Sakha $_{1}$} & $T_{1}$ & $42.63^{\mathrm{f}-\mathrm{j}}$ & $43.00^{\mathrm{a}}$ \\
\hline & $\mathbf{T}_{2}$ & $39.23^{\mathrm{KI}}$ & $39.23^{\mathrm{efg}}$ \\
\hline & $\mathbf{T}_{3}$ & $43.40^{\mathrm{d}-\mathrm{h}}$ & $40.25^{\text {def }}$ \\
\hline & $\mathbf{T}_{4}$ & $41.02^{\mathrm{h}}{ }^{\mathrm{k}}$ & $41.01^{\mathrm{b}-\mathrm{e}}$ \\
\hline & $\mathbf{T}_{5}$ & $41.17^{\mathrm{h}-\mathrm{k}}$ & $40.50^{\text {cde }}$ \\
\hline \multirow{5}{*}{ Sakha $_{2}$} & $\mathbf{T}_{1}$ & $46.00^{\mathrm{abc}}$ & $42.00^{\mathrm{a}-\mathrm{d}}$ \\
\hline & $\mathbf{T}_{2}$ & $40.25^{\mathrm{jk}}$ & $40.17^{\text {def }}$ \\
\hline & $\mathbf{T}_{3}$ & $41.67^{\mathrm{g}-\mathrm{k}}$ & $38.50^{\mathrm{fgh}}$ \\
\hline & $\mathbf{T}_{4}$ & $36.00^{\mathrm{m}}$ & $38.25^{\mathrm{gh}}$ \\
\hline & $\mathbf{T}_{5}$ & $42.75^{\mathrm{f}-\mathrm{J}}$ & $41.37^{\mathrm{a}-\mathrm{d}}$ \\
\hline \multirow{5}{*}{ Sakha $_{3}$} & $\mathbf{T}_{1}$ & $41.00^{\mathrm{h}-\mathrm{k}}$ & $43.10^{\mathrm{a}}$ \\
\hline & $\mathbf{T}_{2}$ & $37.50^{\operatorname{lm}}$ & $34.25^{1}$ \\
\hline & $\mathbf{T}_{3}$ & $33.00^{\mathrm{n}}$ & $42.50^{\mathrm{ab}}$ \\
\hline & $\mathbf{T}_{4}$ & $43.00^{\mathrm{e}-1}$ & $41.25^{\mathrm{a}-\mathrm{d}}$ \\
\hline & $\mathbf{T}_{5}$ & $37.33^{\operatorname{lm}}$ & $37.33 \mathrm{~h}$ \\
\hline \multicolumn{2}{|c|}{ Significant } & $* *$ & $* *$ \\
\hline
\end{tabular}




\section{REFERENCES}

A.O.A.C. (1999). Association of Official Agriculture Chemists, Official Methods of Analysis, $13^{\text {th }}$ Ed., Washington D. C., USA.

Abd El-Motaleb, H.M. and A.M. Gomaa (2004). Yield response of two canola varieties to nitrogen and biofertilizers under sandy soil conditions. Agric. Res. J., Suez Canal Univ., 4 (2): 1-8.

Afridi, M.Z.; M.T. Jan and A.A. Shad (2002). Some aspects of NPK nutrition for improved yield and oil contents of canola. Asian J. Plant. Sci., 1 (5): 507509.

Ahmed, E.B. (2001). Productivity of some sunflower hybrids by using modern system of irrigation and fertilization under newly reclaimed soil. Ph.D. Agron. Dept., Agric., Assiut. Univ., Egypt.

Al-Barrak, K.M. (2006). Irrigation intervals and nitrogen level effects on growth and yield of canola (Brassica napus L.). Theoretical and Applied Genetics, 91 (1): 118-121.

Ali, E.A. and A.E. Hassan (2002). Anatomical structure, chemical constituents, yield and yield components of two rapeseed (Brassica napus L.) cultivars as affected by nitrogen and potassium fertilization. Egypt. J. Appl. Sci., 17 (10): 176-197.

Ali, H.G.; S.K. Nadaf; S. Alkhamisi and A.N. Al-Bakri (2011). Adaptability of canola (Brassica juncea) varieties in different regions of Oman. Int. J. Agric. Bio., 13 (5): 831-834.

Asghar, H.; M. Zahir; Arshad and A. Khaliq (2002). Relationship between in vitro production of auxins by rhizobacteria and their growth promoting activities in Brassicajuncea L. J. Bio. Fertility of soil of Soils; 35 (4): 231-237.
Bagheri, H.; Y. Sharghi and M. Yazdani (2011). The study of planting density on some agronomic traits of spring canola cultivars. Australian. J. Basic and Appl. Sci., 5 (10) :1302-1305.

Duncan, D.B. (1955). Multiple Rang and Multiple F Test, Biometrics, 11: 1-42.

El-Demrdash, I.S. and E.A. Ali (2005). Yield and yield components of some rapeseed (Brassica napus L.) varieties as affected by nitrogen fertilization. The $11^{\text {th }}$ Conference, Agron. Dept., Fac. Agric., Assiut Univ., 471- 477.

Elewa, T.A.; B.B. Mekki; B.A. Bakry and M.F. El-Kramany (2014). Evaluation of some introduced canola (Brassica napus L.) varieties under different nitrogen fertilizer level in newly reclaimed sandy soil. Middle- East J. Sci. Res., 21 (5): 746-755.

Kandil, A.A. (1984). Preliminary study on the effect of NPK fertilization on the oil seed rape (Brassica napus L.). Res. Bull. Fac. of Agric. Zagazig Univ.

Kappen, L.; G. Schultz; T. Gruler and P. Widmoser (2000). Effect of $\mathrm{N}$ fertilization on shoots and roots of rape (Brassica napus L.) and consequences for the soil martic potential. J. Plant Nut. and Soil Sci., 163 (5): 481-489.

Khan, N.; A. Jan; I. Hsanullahb and I.A. Khan (2002). Response of canola to nitrogen and sulphur nutrition.Asian. J. Plant. Sci., 1 (5): 516-518.

Mekki, B.B. (2013). Yield and quality traits of some canola varieties grown in newly reclaimed sandy soils in Egypt. World Appl. Sci. J., 25(2): 258-263.

Mekki, B.B. (2007). The potential of canola quality (Brassica napus L.) as a new winter oil crop in Egypt. Proc. of $12^{\text {th }}$ Int. Conf., Rapeseed Congress sustainable Development Oilseed Crops Production, Wuhan, China, March 2630, 2007. 
Moghadam, H.R.T.; H. Zahedi; F. Ghooshchi and S. Lak (2011). Effect of super absorbent application on destructive oxidative stress in canola (Brassica napus L.) cultivars under water stress conditions. Research on Crops, 12 (2): 393-401.

Pennock, D.; F. Walley; M. Solohub; Si.B and G. Hnatowich (2001). Topographically controlled yield response of canola to nitrogen Fertilizer. Soil. Sci. Sco. Ame. J., 65 (6): 1838-1845.

Richard, L.A. (1954). Diagnosis and Improvement of Saline and Alkali Soils U.S.A. Handbook No. 60 Washington D. C., U.S.A.

Seyed, M.N.S.; I. Allahdadi; S.A. Seyed Mohammadi and E. Sarafraz (2011). Effect of different amount and irrigation intervals on some agronomic characteristics at spring canola varieties
(Brassica napus L.). Int. J. Agric. Sci., 1 (1): 20-31.

Shahin, M.M.; M.M. El-Koliey and M.F. Wahba (2000). Rape seed response to irrigation and nitrogen fertilization. Egyptian. J. Soil. Sci., 40 (1/2): 35- 47.

Sharief, A.E. and M.M. Keshta (2000). Response of some canola cultivars (Brassica napus L.) to different sources and levels of nitrogen fertilizer in soil affected by salinity. Zagazig J. Agric. Res., 27 (3): 603-616.

Snedecor, G.W. and W.G. Cochran (1990). Statistical Methods. $8^{\text {th }}$ Ed. Iowa State Univ. Press Ames, Iowa, USA.

Zarei, G.; H. Shamsi and S.M. Dehghani (2010). The effect of drought stress on yield, yield components and seed oil content to three autumnal rapeseed cultivars (Brassica napus L.). J. Res. Agric. Sci., 6 (1): 29-37.

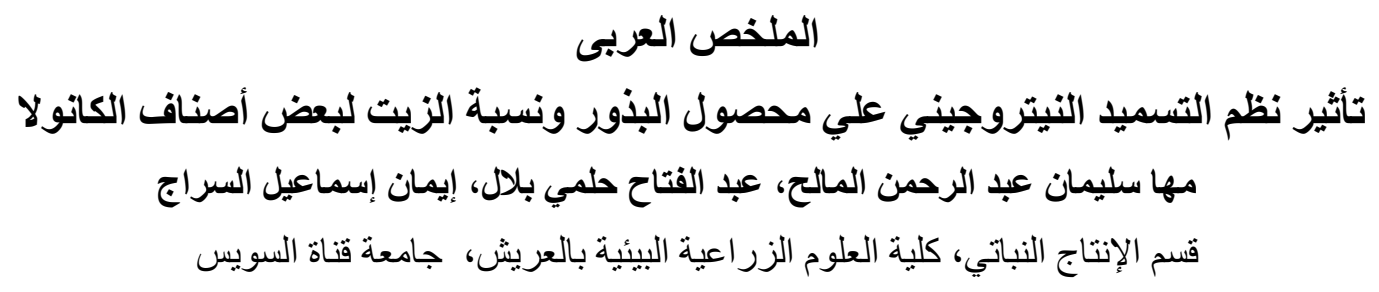

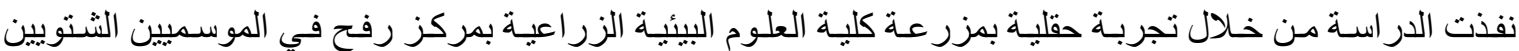

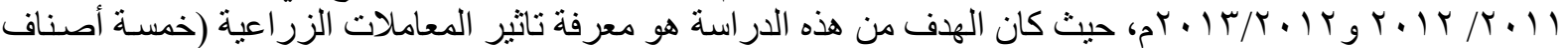

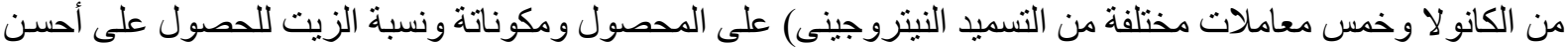

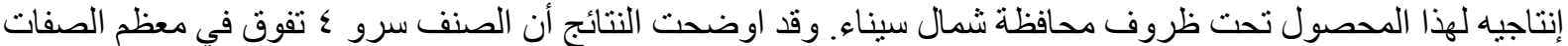

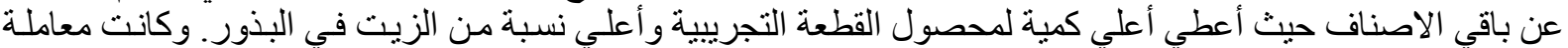

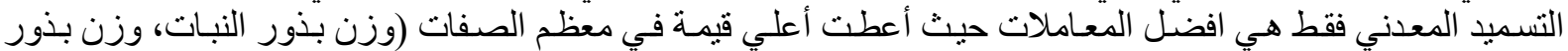

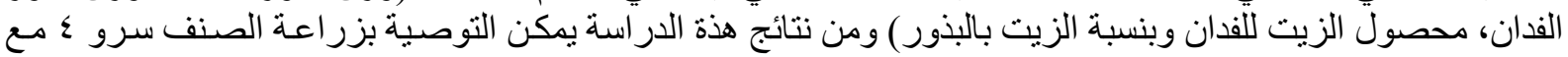
تسميده بالتسميد المعدني فقط للحصول لفئل على افضل النتائج. الكلمات الإسترشادية: الكانو لا، التسميد النيتروجيني، التسميد الحيوي، اصناف الكانو لا، البكتريا المثبتة للنيتروجين، بكتريا الازنوبكتر. 